\title{
Separating the kinetic Sunyaev-Zel'dovich effect from primary cosmic microwave background fluctuations
}

\author{
O. Forni and N. Aghanim \\ IAS - CNRS, Université Paris Sud, Bâtiment 121, 91405 Orsay Cedex, France
}

Received 24 July 2003 / Accepted 22 February 2004

\begin{abstract}
In the present work, we propose a new method aiming at extracting the kinetic Sunyaev-Zel'dovich (KSZ) temperature fluctuations embedded in the primary anisotropies of the cosmic microwave background (CMB). We base our study on simulated maps without noise and we consider very simple and minimal assumptions. Our method essentially takes benefit from the spatial correlation between KSZ and the Compton parameter distribution associated with the thermal Sunyaev-Zel'dovich (TSZ) effect of the galaxy clusters; the latter can be obtained by means of multi-frequency based component separation techniques. We reconstruct the KSZ signal by interpolating the CMB fluctuations without making any hypothesis other than that the CMB fluctuations are Gaussian distributed. We present two ways of estimating the KSZ fluctuations, after the interpolation step. In the first, we use a blind technique based on canonical Principal Component Analysis, while the second uses a minimisation criterion based on the fact that KSZ dominates at small angular scales and that it follows a non-Gaussian distribution. Using the correlation between the input and reconstructed KSZ map we show that the latter can be reconstructed in a very satisfactory manner (average correlation coefficient between 0.62 and 0.90 ), furthermore both the retrieved KSZ power spectrum and temperature fluctuation distribution are in quite good agreement with the original signal. The ratio between the input and reconstructed power spectrum is indeed very close to one up to a multipole $\ell \sim 200$ in the best case. The method presented here can be considered as a promising starting point to identify in CMB observations the temperature fluctuation associated with the KSZ effect.
\end{abstract}

Key words. cosmology: cosmic microwave background - methods: data analysis

\section{Introduction}

The Cosmic Microwave Background (CMB) temperature anisotropies contain the contribution of both the primary cosmological signal, directly related to the initial density fluctuations, and the foreground which include the secondary anisotropies generated after matter-radiation decoupling. They arise from the interaction of the CMB photons with matter and can be of a gravitational type (e.g. Rees-Sciama effect (Rees \& Sciama 1968)), or of a scattering type when the matter is ionised (e.g. Sunyaev-Zel'dovich (SZ) effect (Sunyaev \& Zel'dovich 1972) or Ostriker-Vishniac effect (Ostriker \& Vishniac 1986; Vishniac 1987)). Among all these secondary anisotropies, the dominant effect is the SZ effect. It represents the inverse Compton scattering of the $\mathrm{CMB}$ photons by the free electrons of the ionised and hot intra-cluster gas. It results in the so-called thermal SZ (TSZ) effect whose amplitude is characterised by the Compton parameter $y$ (the integral of the pressure along the line of sight). The TSZ amplitude thus depends only on the cluster electron temperature and density distribution. The inverse Compton effect moves the CMB photons

Send offprint requests to: $\mathrm{O}$. Forni, e-mail: Olivier.Forni@ias.u-psud.fr from the lower to the higher frequencies of the spectrum. This results in a peculiar spectral signature with a decrement at long wavelengths and an increment at short wavelengths. When the galaxy cluster moves with respect to the CMB rest frame, with a peculiar radial velocity $v_{\mathrm{r}}$, the Doppler shift induces an additional effect often called the kinetic SZ (KSZ) effect, which generates temperature anisotropies with the same spectral signature, at least in the non-relativistic approximation, as the primary CMB fluctuations.

The interest of the TSZ effect for cosmology has been recognised very early (see reviews by Rephaeli 1995; Birkinshaw 1999; Carlstrom et al. 2002). It is a powerful tool for detecting high redshift galaxy clusters since it is redshift independent. In combination with X-ray observations it can be used to determine the Hubble constant and probe the intracluster gas distribution. Moreover, the KSZ effect may be the one of the best ways of measuring the cluster peculiar velocities by combining thermal and kinetic effects (Sunyaev \& Zel'dovich 1980). The advantages of this method are: (i) it yields directly the peculiar velocities, bypassing the need to measure inaccurate distance indicators (Faber \& Tully 1976; Tully \& Fisher 1977); (ii) the method has a physical explanation and (iii) it is independent of distance. The KSZ effect can 
be distinguished from the TSZ effect by the different frequency dependence of their intensities. The KSZ intensity reaches its maximum at a frequency of $\sim 218 \mathrm{GHz}$, just where the TSZ intensity is zero. Hence, this is the optimal frequency to the detect the KSZ signal. It has also been shown (Hobson et al. 1998; Bouchet \& Gispert 1999; Baccigalupi et al. 2000; Delabrouille et al. 2003; Kuo et al. 2004; Maisinger et al. 2004) that the TSZ signal can be extracted from the other astrophysical contributions by component separation techniques (Wiener filtering, Maximum Entropy, Independent Component Analysis, ...). Despite the scientific interest of the KSZ effect as a probe of large scale matter distribution and structure formation theories, very few measurements of the peculiar velocities are achieved (Holzapfel et al. 1997; Lamarre et al. 1998; Benson et al. 2003). As a consequence, very few methods have been proposed so far to address the specific underlying question of how to separate the secondary KSZ fluctuations from the primary anisotropies. In an early work, Haehnelt \& Tegmark (1996) used an optimal filtering (Wiener), with a spatial filter derived from X-ray observations of galaxy clusters, that minimises the confusion with CMB. However, this method implied knowledge of the CMB power spectrum. Aghanim et al. (1997) rather used a matched filter optimised on simulated data and independent of the underlying CMB model. Recently Hobson \& McLachlan (2003) presented a Bayesian approach for detecting and characterising the signal from discrete objects embedded in a diffuse background. They showed that this approach is around twice as sensitive as the linear optimal filter approach proposed by Haehnelt \& Tegmark (1996).

In the present study, we propose a new method optimised to extract from the primary anisotropies the temperature fluctuations associated with the KSZ effect. The method is based on the fact that we have two sets of maps (provided, in a realistic case, by component separation techniques); the first set contains both $\mathrm{CMB}$ and $\mathrm{KSZ}$ temperature fluctuations and the second set consists of Compton parameter maps associated with the TSZ effect which is used as a spatial template. In our study, we do not use real (observed) maps but rather two sets of simulated maps. We were able to retrieve, in the best possible way, the amplitude and the distribution of the temperature fluctuations associated with KSZ together with the associated power spectrum.

\section{Methodology}

In a "real-life" case, it is worth noting that the application of the method we propose here is based on the fact that a first-step component separation is performed on the CMB data, leaving us with a TSZ effect map and a temperature fluctuation map containing primary and KSZ anisotropies. In the present study, we focus on the description of the method and the way it is intrinsically limited by the pure cosmological signals: primary $\mathrm{CMB}+\mathrm{SZ}$ effect (without adding any instrumental effects). It is beyond the scope of this first study to address the instrumental effects (this will be the subject of a future work), therefore and as mentioned above, we use simulated cosmological data. Namely, we simulate $15(512 \times 512$ pixels $)$ primary $\mathrm{CMB}, \mathrm{TSZ}\left(\right.$ mean $\sigma_{\mathrm{y}}=1.17 \times 10^{-5}$ ) and KSZ maps (mean $\sigma_{\mathrm{KSZ}}=1.85 \times 10^{-6}$ ) with a pixel size of 1.5 arc-min. A precise description of the $\mathrm{SZ}$ simulations is given in Aghanim et al. (2001). The KSZ effect induces temperature fluctuations that can be written as $\delta_{\mathrm{T}}^{\mathrm{KSZ}}=(\Delta T / T)_{\mathrm{KSZ}}=-\frac{v_{\mathrm{r}}}{c} \tau$, with $c$ and $\tau$ the velocity of light and the cluster Thomson optical depth. The primary CMB and the KSZ anisotropies having the same spectral shape in the non-relativistic approximation, we construct maps of thermodynamic temperature fluctuations, $\delta_{\mathrm{T}}$, by adding the two signals $\delta_{\mathrm{T}}=(\Delta T / T)_{\mathrm{KSZ}}+(\Delta T / T)_{\mathrm{CMB}}$. We are thus left with two data sets of pure cosmological signals, one consisting of the temperature fluctuation maps $(\mathrm{CMB}+\mathrm{KSZ})$ and the other consisting of the Compton parameter maps, $y$, for the TSZ effect. For this study, we adopt a low matter density flat model defined by: $\Omega_{\mathrm{m}}=0.3, \Omega_{\Lambda}=0.7$ and $h=H_{0} / 100 \mathrm{~km} \mathrm{~s}^{-1} \mathrm{Mpc}^{-1}=0.65$.

Once the two types of map are provided, $y$ maps for the TSZ effect and $\delta_{\mathrm{T}}$ maps for CMB $+\mathrm{KSZ}$, our goal is to obtain the best possible estimate of the KSZ. To achieve this goal, we benefit from the fact that the TSZ and the KSZ features are spatially correlated, as already noted by Diaferio et al. (2000) and Sorel et al. (2002). The latter showed that the absolute values of the covariance coefficients between TSZ and KSZ maps are significantly high even though the correlation coefficient between the maps does not exceed 0.1 in absolute value. This low value is due to the fact the signs and amplitudes of the KSZ anisotropies in a map depend on the distribution of the radial peculiar velocities, which is a random variable with zero mean. The spatial correlation between TSZ and KSZ simply means that both effects are due to galaxy clusters. Therefore, where the TSZ signal is present so are the KSZ fluctuations regardless of their signs or amplitudes. Conversely, where the TSZ fluctuations are absent, so are the KSZ fluctuations and the signal at that position in the $\delta_{\mathrm{T}}$ map is therefore associated with the CMB anisotropies only.

Our technique to separate the KSZ fluctuations from the primary CMB anisotropies is based on this simple statement. It allows us to build up a two-step strategy in which: (i) we first derive the best estimate of the CMB map, and (ii) consequently deduce the best estimate of the KSZ map.

\subsection{Estimating the primary $C M B$ anisotropies}

In this section, we address the first step of our separation method, namely we derive an estimate (the best possible) of the primary fluctuation map. For this we use the two observables: the TSZ map and the $\delta_{\mathrm{T}}$ map which contains both the primary and the KSZ fluctuations. Given the above mentioned statement on the spatial correlation between TSZ and KSZ signals, the basic idea in order to estimate the primary CMB anisotropies, is to use the TSZ map as a mask to select in the $\delta_{\mathrm{T}}$ map, the pixels where the TSZ fluctuations are not present, i.e. where only primary anisotropies are present. The rest of the pixels in the map are missing or masked pixels. We then interpolate the $\delta_{\mathrm{T}}$ signal on these missing pixels with the constraint that the pixels where TSZ is absent i.e. with signal associated with primary CMB only, keep their values after the interpolation is achieved. We therefore end up with an estimated primary 
CMB map where the $\delta_{\mathrm{T}}$ signal in the masked (missing) pixels is obtained from the interpolation. Formally, the KSZ map can then be estimated simply by computing the difference between the original unmasked $\delta_{\mathrm{T}}$ map and the primary CMB map estimated with the interpolation.

\subsubsection{Interpolation}

We already note that the "recovery" of the KSZ map heavily relies on the performances of the interpolation method. We first use the method described in Unser (1995). Consider the problem of the minimisation of a general criterion written as:

$$
\begin{aligned}
E(u)= & \sum_{(k, l) \in \mathbb{Z}^{2}} w(k, l)[f(k, l)-u(k, l)]^{2} \\
& +\lambda \sum_{(k, l) \in \mathbb{Z}^{2}}\left[d_{x} * u(k, l)\right]^{2}+\left[d_{y} * u(k, l)\right]^{2}
\end{aligned}
$$

where $f$ is an input image, $u$ is the desired solution $w \geqq 0$ is a map of space-varying weights, $d_{x}$ and $d_{y}$ are the horizontal and vertical gradient operators, respectively. The second spaceinvariant term in Eq. (1) is a membrane spline regulariser; the amount of smoothness is controlled by the parameter $\lambda$. Taking the partial derivative of Eq. (1) with respect to $u$, we find that $u$ is the solution of the differential equation:

$f_{w}=W u+\lambda L u=A u$

where $W$ is the diagonal weight matrix, $f_{w}=W f$ the weighted data vector, $L$ is the discrete Laplacian operator and $A=$ $W+\lambda L$ a symmetric definite matrix. The inversion of Eq. (2) is achieved using a multi-grid technique (Wesseling 1992). Typically, we need two V-cycles with two iterations in the smoothing Gauss-Seidel part of the algorithm to reach a residual of the order of $10^{-6}$. In our case, the interpolation of the primary CMB map is achieved by setting the weights to zero where the data are missing, i.e. in the masked pixels, and to one elsewhere and by solving Eq. (2). The value of $\lambda$ then determines the tightness of the fit at the known data points (unmasked pixels), while the surface $u$ is interpolated such that the values of the Laplacian of $u$ are zero elsewhere. In the present work, we impose a low value for $\lambda$ so that the recovered values at the known data points are equal to the original values. This criterion can be relaxed to take into account corruption of the data by additive white noise (Unser 1995). In this case, the optimum regularisation parameter $\lambda$ can be defined as:

$$
\lambda=\frac{\sigma^{2}}{E(f . L f)-4 \sigma^{2}}
$$

where $\sigma^{2}$ is the variance of the noise, and $E(f . L f)$ denotes an estimate of the correlation between the noisy image $f$ and its Laplacian $L f$. In the other cases (non-white noise), the optimal regularisation parameter $\lambda$ may be determined from the data using cross-validation methods (Wahba 1977), or from a given measurement model of the signal + noise (Reeves 1994).

It is possible to improve the performance of the interpolation, and hence of the retrieved KSZ map, by setting nonzero values to the Laplacian of $u$ at the missing data points (which are set to zero in the original method). The values we set for the Laplacian of $u$ are such that the first and second derivatives of the interpolated signal are continuous throughout the interval. These continuity conditions characterise the cubic B-spline functions which are known for their simplicity and their performance in terms of signal reconstruction (Unser et al. 1993; Thévenaz et al. 2000). In practice, these additional conditions imply that the source term $f_{w}$ in Eq. (2) is modified to impose non-zero values at the points where the weights are set to zero (i.e. the missing data points). An equivalent way to solve Eq. (2) with the above mentioned conditions is to replace the Laplacian operator $L$ by the quadratic operator $L^{2}$. These two interpolation methods are obviously not unique, and other techniques (based on textures for example) can be used. In the following we will only test the two operators described above and then choose among them the one that gives the most satisfying results.

\subsubsection{Defining the mask or missing pixels}

We must now define more precisely what we mean when we state where the TSZ is not present; or in other terms how do we select the missing data points? Besides the pixels that actually contain no galaxy clusters, i.e. no SZ contributions, this statement means that we fix a threshold value for the TSZ amplitude below which we consider that the TSZ signal is too small to be detected. The corresponding pixels in the $\delta_{\mathrm{T}}$ maps are then considered to be associated only with the primary CMB signal. On the contrary, above this threshold the corresponding pixels in the $\delta_{\mathrm{T}}$ map are considered to be the missing data points, i.e. masked pixels that we want to interpolate. It is clear that the number and location of the missing data will depend on the threshold. The lower it is, the larger the number of missing data we need to recover. The choice of this threshold also has important consequences for the quality of the interpolation.

When the threshold is high, on the one hand, the number of missing data is small and the interpolated surface is good. On the other hand, the selection retains only the clusters with the highest TSZ and misses the majority of clusters. In this case, we expect to end up with a low correlation coefficient between the retrieved and the original KSZ maps. When the threshold is low, we take into account a majority of clusters, but the interpolated surfaces are large and the quality of the interpolation suffers from that. Moreover, the characteristic scale of the interpolated surfaces becomes, in this case, of the order of that of the CMB fluctuations, leading to "confusion effects" in the interpolation. From these remarks, we can infer that: firstly, there will exist an optimal threshold value for which the correlation between the retrieved and the original KSZ maps is maximum; secondly, the restoration of extended clusters is likely to be of low quality, as already noted by Haehnelt \& Tegmark (1996).

Obviously there is no a priori way to choose the TSZ threshold on an objective basis. Indeed, given that the TSZ map is obtained (in "real-life") from a component separation process involving the true signal but also the instrumental and observational effects, one cannot rely on a "theoretical" expected value. Therefore, rather than performing only one 
interpolation of the primary CMB map for one single TSZ threshold, we propose to retrieve a set of interpolated CMB maps corresponding to a set of TSZ threshold values. The later can be defined in a simple way without any theoretical or observational prior as follows: we compute the cumulative distribution function of the TSZ values in the given map and we search for the values corresponding to from $5 \%$ to $95 \%$ of the total number of pixels (with a step of $5 \%$ ). This gives us a set of 19 threshold values such that all pixels in the TSZ map that have $y$ parameters above the threshold are identified as the missing data points in the simulated $\delta_{\mathrm{T}}$ map, i.e. the mask. In the present study, we are using simulated TSZ maps, i.e. without noise. These maps exhibit a background of zero values, which proportion represents in our case at least $10 \%$ of the total pixel number. This characteristics implies that the first two TSZ threshold values associated with 5 and $10 \%$ of the total pixel number are irrelevant. In the following, we thus use only the 17 highest TSZ thresholds.

\subsubsection{Results}

For each of the 15 simulated maps, we obtain 17 TSZ threshold values, and thus 17 masked $\delta_{\mathrm{T}}$ maps. We apply the interpolation techniques (Sect. 2.1.1) to recover the primary CMB signal in the masked regions. For each of the 15 simulated maps, we thus end up with 17 estimated primary CMB maps corresponding to the 17 TSZ threshold values. The associated KSZ maps are evaluated simply by subtracting the interpolated primary CMB maps from the total $\delta_{\mathrm{T}}$ map.

To evaluate how well the two interpolation methods presented in Sect. 2.1.1 do recover the KSZ signal, we compute for each of the $17 \mathrm{KSZ}$ estimated maps the correlation coefficient between the original input KSZ map and the estimated KSZ maps. The results are shown in Fig. 1. The data points representing the correlation coefficients are plotted as a function of the standard deviation of the estimated KSZ map for each of the 17 threshold values. The diamonds and the dashed line represent the case where the interpolation is such that the Laplacian values are set to zero, and the triangles and the solid line are for the case in which the Laplacian values are nonzero. The upper panel in Fig. 1 shows our best recovery case in terms of correlation coefficient. The lower panel is for our worst case. The correlation coefficients between the original input KSZ map and the 17 estimated KSZ maps are also displayed as a function of the 17 TSZ threshold values in Fig. 2. It is worth noting that the high TSZ thresholds (abscissae in Fig. 2) correspond to low KSZ standard deviations (abscissae in Fig. 1).

First, we note from Fig. 1 that, for any standard deviation of the estimated KSZ map, the correlation coefficient between the original and the estimated KSZ maps is higher when the Laplacian values are non-zero than when they are set to zero. Actually there can be a significant improvement in the KSZ reconstruction if an optimised interpolation method is used. This is especially true for the maps with low standard deviations. The improvement brought by the biharmonic operator is of the order of $20 \%$ in our worst case (Fig. 1, lower panel). We will
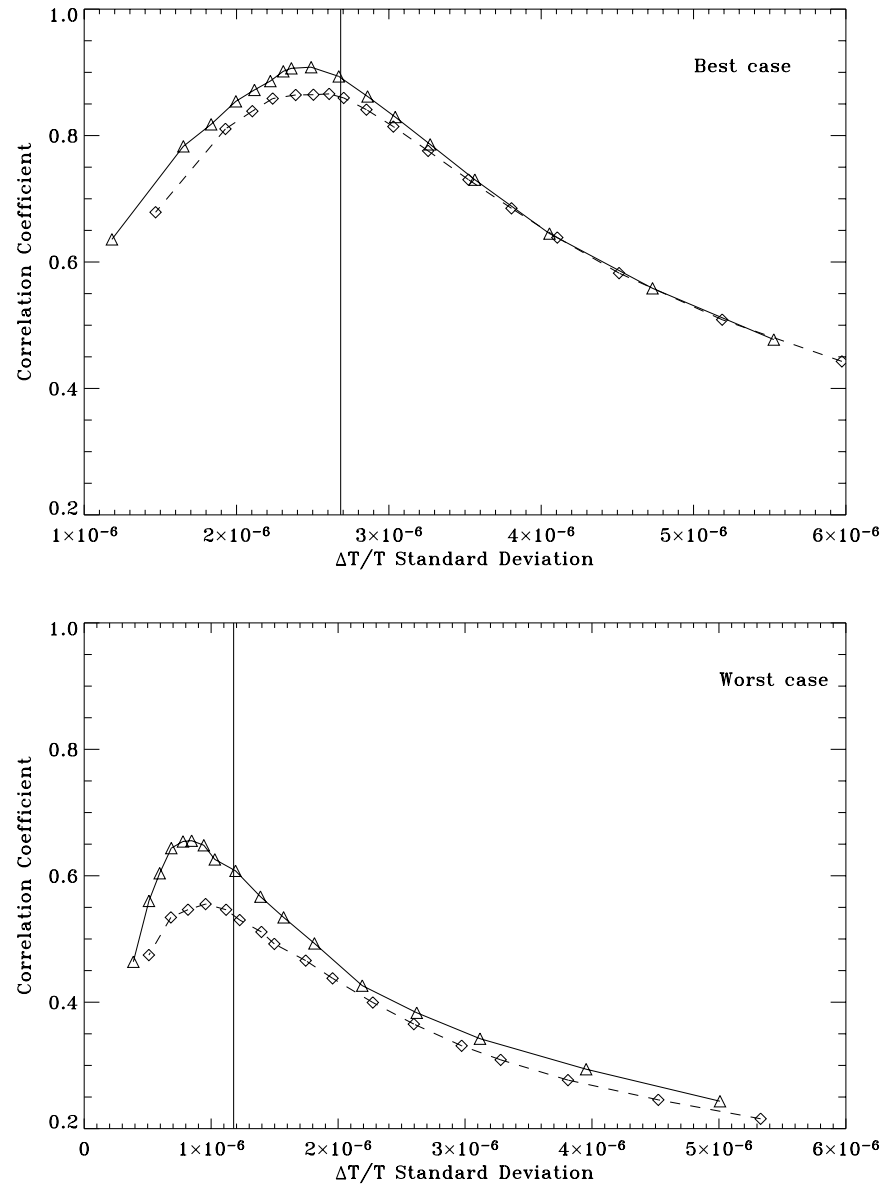

Fig. 1. The correlation coefficient between the original KSZ map and the series of 17 estimated KSZ maps as a function of the standard deviations of the estimated KSZ maps. Upper panel is for the best case and lower panel for the worst case. The triangles and the solid line stand for the interpolation with the biharmonic operator, the diamonds and the dashed line are for the interpolation with the Laplacian. The interpolation with the biharmonic operator gives better results, especially for the KSZ maps with low standard deviation. The vertical lines mark the standard deviation of the original $\mathrm{KSZ}$ maps $\left(2.6 \times 10^{-6}\right.$ and $\left.1.2 \times 10^{-6}\right)$. The standard deviation of the primary $\mathrm{CMB}$ is $1.9 \times 10^{-5}$.

therefore use, in the following, the most powerful interpolation method, which is the one with the $L^{2}$ operator.

Second, as expected, the correlation coefficient increases when the TSZ threshold decreases as shown in Fig. 2 (i.e. when the standard deviation of the estimated KSZ map increases in Fig. 1). The correlation coefficient reaches a maximum value and then it decreases for the lowest TSZ thresholds (i.e. the highest KSZ standard deviations). Moreover, we note that among the set of $17 \mathrm{KSZ}$ estimated maps the one with the highest correlation coefficient is shifted towards lower values proportionally to the standard deviation of the original KSZ map. We will use this behaviour later on in the minimisation procedure. 


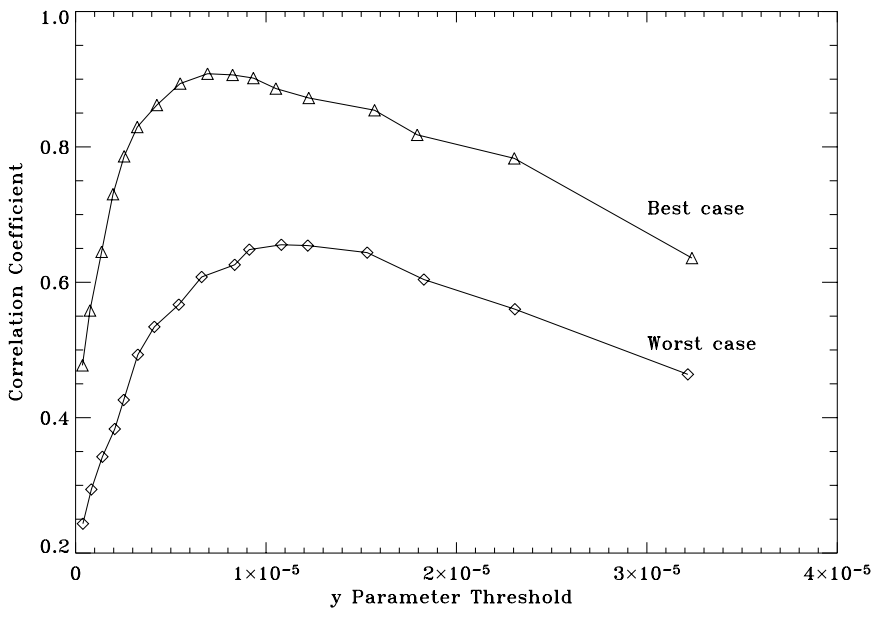

Fig. 2. For the best case (upper curve and triangles) and worst case (lower curve and diamonds), the correlation coefficient between the original and the 17 estimated KSZ maps as a function of the associated $17 \mathrm{TSZ}$ threshold values. The highest threshold value is of the order of $y=3.5 \times 10^{-5}$. The interpolation method uses the biharmonic operator.

\subsection{Reconstructing the $K S Z$ map}

In the previous step, we interpolated the $\delta_{\mathrm{T}}$ signal to estimate the primary $\mathrm{CMB}$ map and then extract the KSZ signal as a function of a set of TSZ thresholds. We tested the performances of two interpolation methods by comparing through a correlation coefficient each of the 17 estimated KSZ maps, corresponding to the $17 \mathrm{TSZ}$ threshold values, to the original input KSZ map which, of course, we do not have in "real life". The set of 17 estimated KSZ maps were obtained by subtracting the interpolated primary CMB maps from the total $\delta_{\mathrm{T}}$ map of the temperature fluctuations.

In this step, using the set of $17 \mathrm{KSZ}$ estimated maps associated with the set of 17 TSZ thresholds, we search for a method that gives us either the reconstructed KSZ map which is the closest to the original KSZ signal or even better, the combination of the set of KSZ maps giving the best estimate of the original KSZ map. In the following, we have explored two ways to achieve this goal (restricted to linear combinations only). The first way is to decorrelate the set of images by a canonical Principal Component Analysis (PCA), the second is to minimise a criterion, which in our case is related to the nonGaussian character of the KSZ signal.

\subsubsection{Decorrelation with principal component analysis}

It is obvious from our definition of the masked pixels (Sect. 2.1.2) that all the interpolated maps (defined by the set of 17 TSZ thresholds) are highly correlated. The first and natural approach to decorrelate them is thus to use a PCA method. As noted in Sect. 2.1.2, decreasing the TSZ threshold increases the characteristic scale of the structures we have to interpolate. This means that the confusion between the extended clusters and the primary CMB anisotropies increases. As a consequence, decreasing the TSZ threshold increases the proportion of the $\delta_{\mathrm{T}}$ signal due to the primary CMB in the estimated
KSZ maps. In this context, the purpose of performing a PCA is to decorrelate the signal due to the primary CMB from that due to the galaxy clusters. We expect to find the high frequency part of the KSZ effect in one principal component, and, in a second principal component, the low frequency part of the KSZ signal (essentially the extended clusters) together with the contribution from the primary CMB fluctuations.

For each of the 15 simulated maps, we apply the PCA to the set of 17 estimated KSZ maps. We find that the first and second principal components represent respectively $\sim 70 \%$ and $\sim 15 \%$ of the total input signal. In our approach, it is the first principal component which stands for the KSZ reconstructed signal. It is thus interesting to evaluate how well the PCA performs the reconstruction. To do so, we compute for each of the 15 simulated maps the correlation coefficient between the first principal component and the original input KSZ map. The correlation coefficient averaged over the 15 maps reaches 0.73 which is satisfactory. However, the standard deviation of the first principal component is on average smaller by almost $50 \%$ than the standard deviation of the original KSZ signal. The PCA method clearly underestimates the reconstructed KSZ signal, which is an obvious weakness of the method. In the following we will thus investigate minimisation methods.

\subsubsection{Statistical minimisation}

By minimising on the known KSZ signal (from our dataset of 15 input maps), we can first search for a linear combination of the set of 17 estimated KSZ maps that is the closest to each original KSZ in the sense of least squares. This has been done using a standard singular value decomposition (Press et al. 1992). We compute again the correlation coefficient between the original KSZ map and the reconstructed map obtained from the minimisation to estimate the power of the method. We find an average correlation coefficient (over the 15 simulated input maps) of 0.8 , only slightly higher than the PCA result of 0.73 . However, the standard deviations of the reconstructed maps are again significantly lower than that of the original KSZ maps by almost $25 \%$ on average (better than in the PCA case). Furthermore, the results of the least squares minimisation depend strongly on the set of estimated maps that is used, which is clearly undesirable.

To avoid this problem and to obtain as more mapindependent results as possible, we must identify a trustworthy criterion to minimise on. The latter should ideally give at the same time a result that is the closest possible to the largest correlation coefficient of 0.80 on average (obtained with the least squares minimisation), and reconstructed KSZ maps with standard deviations as close as possible to those of the original KSZ signal. Moreover, a good minimisation criterion would be a criterion that characterises the KSZ signal only, excluding the primary CMB signatures. We have identified two properties of the KSZ fluctuations that fulfill this definition:

- the KSZ signal dominates the primary CMB at high wave numbers (small angular scales);

- the KSZ effect is a highly non-Gaussian process contrary to the primary CMB which is a Gaussian process. 
Table 1. The statistical properties of the first scale ( 3 arc-min) diagonal wavelet coefficients distribution for the $\delta_{\mathrm{T}}$ map (KSZ + CMB), the KSZ map and the primary CMB alone. The two cases stand for our best case (first pair) and worst case (second pair). We note that the three moments are almost identical and characterise well the KSZ fluctuations; they are very different from the CMB fluctuation properties.

\begin{tabular}{c|ccc}
\hline \hline & Standard deviation & Skewness & Excess kurtosis \\
\hline KSZ+CMB & $6.45 \times 10^{-7}$ & 0.10 & 8.71 \\
KSZ & $6.45 \times 10^{-7}$ & 0.10 & 8.72 \\
\hline KSZ+CMB & $2.05 \times 10^{-7}$ & 0.22 & 8.97 \\
KSZ & $2.09 \times 10^{-7}$ & 0.23 & 9.15 \\
\hline $\mathrm{CMB}$ & $1.60 \times 10^{-8}$ & -0.02 & 0.45 \\
\hline
\end{tabular}

The analyses of the available CMB data (Cayon et al. 2003; Komatsu et al. 2003; Santos et al. 2003) all seem to agree on the fact that primary CMB anisotropies are Gaussian distributed as expected from the simplest inflationary models. By contrast, the SZ effect is definitely characterised by its nonGaussian signatures. Using wavelet analysis, we have demonstrated (Aghanim \& Forni 1999; Forni \& Aghanim 1999), that the excess kurtosis of the wavelet coefficients allows us to discriminate between a Gaussian primary CMB signal and a non-Gaussian process like the SZ effect. We note though that a recent re-analysis of the WMAP data (Vielva et al. 2003) suggests that the observed CMB anisotropies exhibit non-Gaussian signatures. The analysis indicates that the deviations from Gaussianity concern large scales (a few degrees). Such a behaviour if confirmed would therefore not affect our minimisation criterion since the latter is based on the characteristics of the signal at small angular scales (a few to a few tens of arcminutes). In particular, the statistical properties of the wavelet coefficients at the lowest decomposition scale (3 arc-min) reflect the properties of the SZ effect only. This is due to our choice of the wavelet basis and of the decomposition scheme which focus on the scale where the SZ signal dominates over the primary CMB. Within this choice, the wavelet analysis provides us with the wavelet coefficients associated with diagonal, vertical and horizontal details in the analysed map. Finally, we have shown in Aghanim \& Forni (1999) that in the case of the SZ effect the diagonal details are by far the most sensitive to the non-Gaussian signatures (recently confirmed and explained by Starck et al. 2004).

In Table 1, we compare, using the 9/7 bi-orthogonal filter bank of Cohen et al. (1990) and for our worst and best cases the statistical properties (standard deviation, skewness and excess kurtosis) of the diagonal details of the KSZ maps and $\mathrm{CMB}+\mathrm{KSZ}$ maps at the first decomposition scale ( 3 arc-min). We also give the values of the three quantities for the primary CMB maps. We immediately note that the two sets of wavelet coefficients for KSZ and KSZ + CMB share the same statistical properties and are quite different from those of the primary CMB alone. This confirms not only that the KSZ signal dominates over the primary CMB (same standard deviation, i.e. same power), but also that the non-Gaussian signatures in the KSZ + CMB maps are associated with the KSZ effect (same skewness and excess kurtosis). The above mentioned properties characterise, in a mixture of $\mathrm{CMB}+\mathrm{KSZ}$ fluctuations, the KSZ effect only. Consequently, we can confidently minimise on them. In practice, we choose the following criterion:

$$
\zeta=\operatorname{Min}\left[\frac{\left(\mathcal{M}_{2}\left(w_{0}\right)-\mathcal{M}_{2}(w)\right)^{2}}{\mathcal{M}_{2}^{2}\left(w_{0}\right)}+\frac{\left(\mathcal{M}_{4}\left(w_{0}\right)-\mathcal{M}_{4}(w)\right)^{2}}{\mathcal{M}_{4}^{2}\left(w_{0}\right)}\right]
$$

where $w_{0}$ is the distribution of the diagonal wavelet coefficients for the known $\delta_{\mathrm{T}}$ map $(\mathrm{KSZ}+\mathrm{CMB})$ and $w$ is the distribution of the diagonal wavelet coefficients for the desired solution map. $\mathcal{M}_{2}$ and $\mathcal{M}_{4}$ are respectively the second and the fourth moments of the wavelet coefficients. This criterion has thus the advantage of taking into account both the energy (or power) content of the coefficients, through the second moment, and the non-Gaussian character, through the fourth moment. We have chosen the fourth moment to characterise the non-Gaussian property because it is the moment for which the KSZ signal is the most sensitive to non-Gaussianity as shown by the hydro-dynamical simulations of da Silva et al. (2001). Clearly, we might also include the third moment of the wavelet coefficients in the criterion. This would be needed in particular if we were dealing with a "skewed" signal such as distorted CMB anisotropies by the weak lensing of large scale structures. Taking the fourth moment in the minimisation criterion allows us in turn to focus on the reconstruction of KSZ maps excluding the signal that might contribute at that particular angular scale. In our minimisation criterion, we have added the second and fourth moments quadratically. We have thus attributed equal weights to the power and to the non-Gaussian character of the KSZ signal. It is possible to envisage a different weighting of one term or the other in Eq. (4). This would in principle enhance the non-Gaussian signal, for example, and thus ease its separation from a Gaussian signal. Such a non-quadratic mixture would be particularly useful at scales where the KSZ effect is not the dominant process. However, there is a priori no trivial way of setting the weights. This possibility should be investigated in the future.

The solution map $w$ is obtained by minimisation of the criterion $\zeta$ over all the combinations of wavelet coefficients $w_{0}$ allowed by our set of 17 estimated KSZ maps (there are 15 simulated input maps). However, there are far too many combinations and we therefore choose to reduce the number of cases. To do so, we recall the observation made in the previous section that the correlation coefficients between the original KSZ map and the 17 estimated KSZ maps exhibit a maximum value (see Fig. 1). To reduce the number of combinations for the 


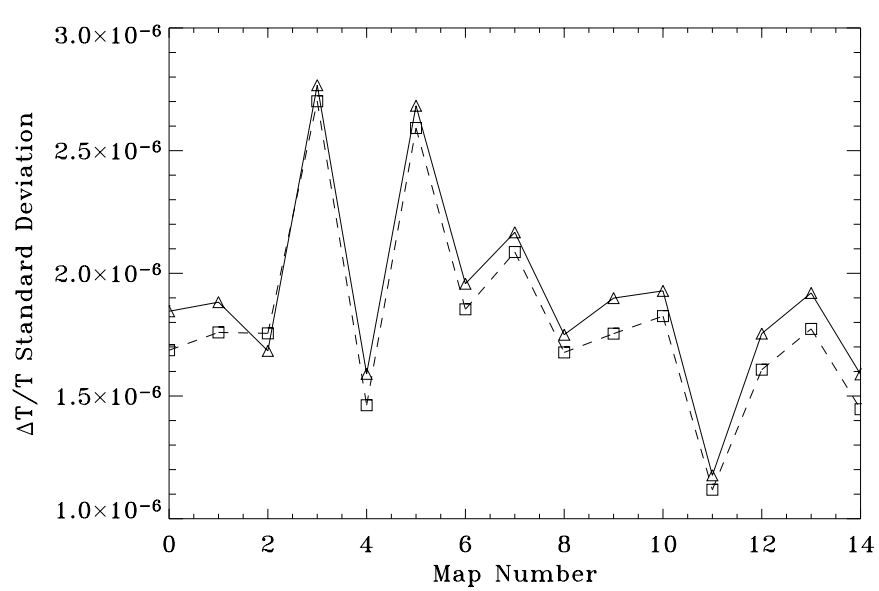

Fig. 3. Standard deviations of our set of $15 \mathrm{KSZ}$ original simulated maps (triangles) as compared to the standard deviations of the 15 reconstructed KSZ maps (squares). The reconstruction is based on the minimisation of the statistical criterion (see text).

minimisation, we then adopt the following iterative strategy: at each step, we first eliminate the estimated KSZ map which contains the highest contribution from the $\mathrm{CMB}$, i.e. the one with the highest standard deviation. Then we minimise $\zeta$ using the remaining set of estimated maps. Finally, we take as a solution of the minimisation criterion the map corresponding to its lowest value.

In addition to the previous conditions (power and nonGaussian character), we also make use in the minimisation process of a nice property of the wavelet transform, which is that it preserves the spatial information. In our case, this means that we can identify the diagonal wavelet coefficients that are spatially associated with the clusters in the TSZ map. Thus instead of minimising over all the wavelet coefficients of the data map $\left(w_{0}\right.$ in Eq. (4)), we can minimise only over the coefficients corresponding to the clusters. This has two advantages; the first is to enhance the non-Gaussian character and the second is to reduce the influence of other possible non-Gaussian processes that could affect the anisotropy map $\delta_{\mathrm{T}}$.

In Fig. 3, we present the standard deviations of the 15 original simulated KSZ maps (triangles) and of the 15 reconstructed KSZ maps (squares) obtained by the minimisation technique described above. The agreement is pretty good even for the maps with the lowest standard deviations. We find that the error on the standard deviation is only of the order of $\sim 5 \%$. This is much smaller than what was obtained from the PCA method $(\sim 50 \%)$ or from the least squares minimisation method $(\sim 25 \%)$. Furthermore, the mean value (over the 15 original maps) of the correlation coefficient between the original and the reconstructed KSZ maps is 0.78 . It is slightly better than the value obtained with the PCA method and quite close to the value of 0.80 obtained with the least squares method. The quality of the KSZ map reconstruction can be observed in Figs. 4 and 5 which display, for our best and worst cases respectively, the histograms of the temperature fluctuations and the power spectra of both the original (solid line) and reconstructed (dashed line) KSZ maps as well as the ratio of these two power spectra. Note that the ratio is close to
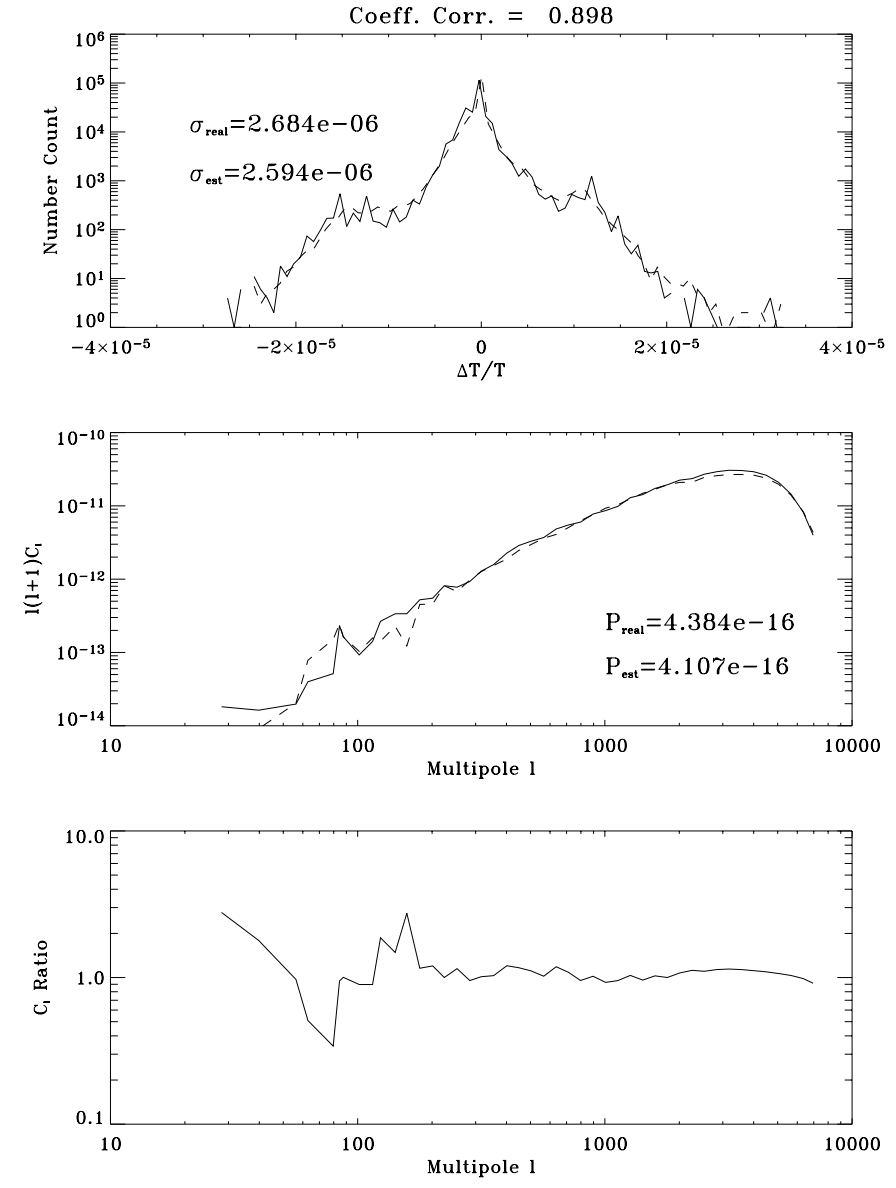

Fig. 4. Top and middle panels: histogram and power spectrum of the original KSZ map (solid line), and of the reconstructed KSZ map (dashed line). The reconstruction is based on the minimisation of the statistical criterion (see text). The bottom panel exhibits the ratio of the two power spectra. Note the correlation coefficient between the original and reconstructed KSZ maps of $\sim 0.9$ and the total power $P_{\text {real }}$ and $P_{\text {est }}$.

one over a large range of multipoles (angular scales) even in the domain where the primary CMB dominates the KSZ signal by orders of magnitude. We also notice the correlation coefficients between the original and the reconstructed KSZ maps which reaches $\sim 0.9$ in our best case and 0.62 in our worst case. The comparison between the standard deviations of the original and the reconstructed map $\sigma_{\text {real }}$ and $\sigma_{\text {est }}$ also gives a global indication on how well the method works. Clearly, the method we propose to separate the KSZ signal from the primary CMB anisotropies despite their identical frequency dependence allows us to obtain such results because we were not only able to estimate correctly the amplitude of the KSZ signal for most clusters but also their angular separation as well as the amplitude of the background (primary CMB). This is nicely exhibited by the superposition of the cuts across the reconstructed (dashed line) and the original (solid line) KSZ maps, once again for the best and worst cases (Figs. 6 and 7 respectively). The method partially fails to find broad KSZ features due to their significant level of confusion with the primary CMB fluctuations (Sect. 2.1.2). Moreover, since the minimisation process is an overall procedure, it can occasionally happen that relatively 

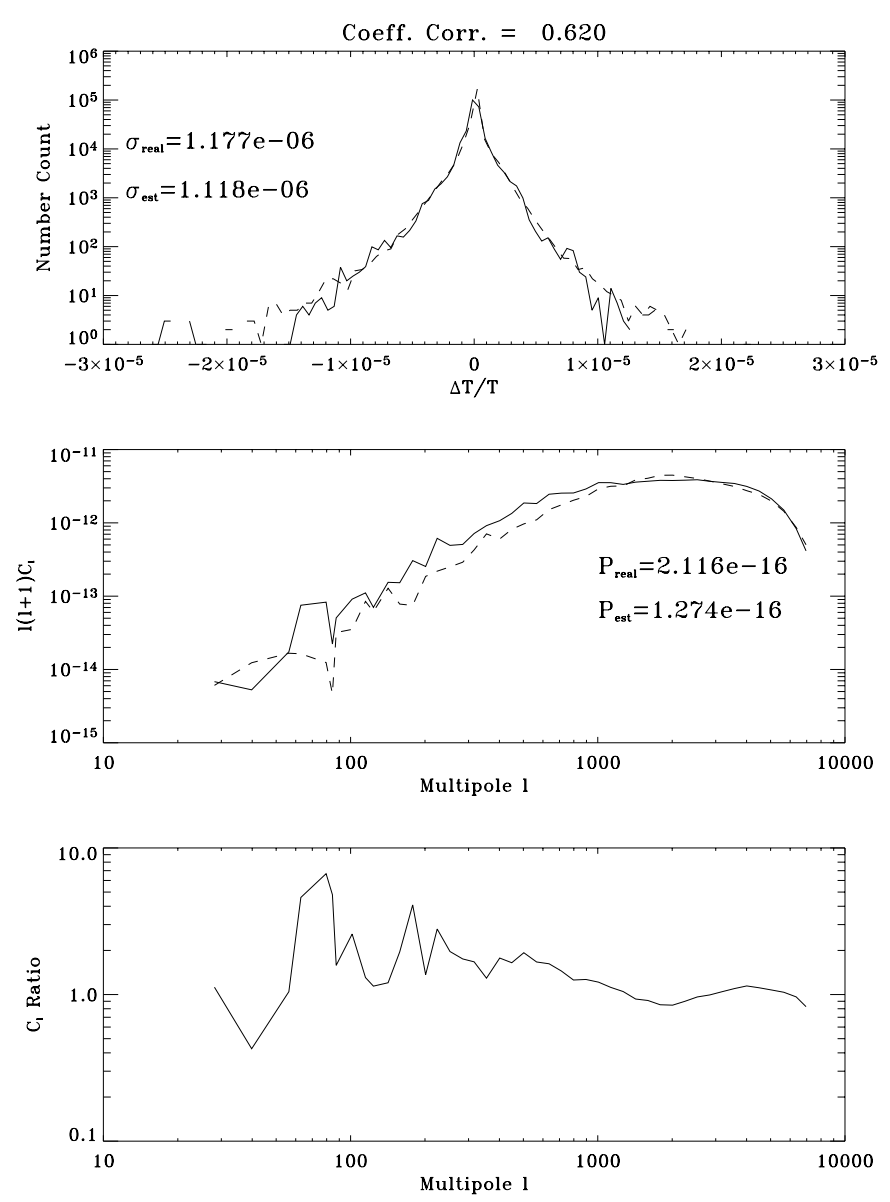

Fig. 5. Same as Fig. 4. This is our worst case and corresponds to the original KSZ map with the lowest standard deviation. Note the low correlation coefficient 0.62 .

large features (i.e. of the order of $10^{-5}$ in absolute $\Delta T / T$ ) are poorly recovered.

\section{Sensitivity test}

We have tested our method to separate the KSZ anisotropies from the primary CMB signal on simulated maps free of any noise. Moreover, we have not taken into account other astrophysical contributions than the CMB and the SZ effect themselves. In "real life", the data are corrupted by instrumental noise and astrophysical signals. Additional noise (whatever its origin) will have a first effect of reducing the ratio between the primary $\mathrm{CMB}$ and the KSZ signals. We test the performance of our method to this effect by applying our procedure to the same KSZ map that is added to the same primary CMB map. The standard deviation of the KSZ signal is reduced while the $\mathrm{CMB}$ standard deviation is kept the same. This results in lowering the KSZ contribution to the $\delta_{\mathrm{T}}$ map. We arbitrarily choose to reduce the standard deviation following a geometrical progression $\sigma_{i}=\sigma_{0} \sqrt{2}^{i}$ with $i=0,6$ and $\sigma_{0}=2.5 \times 10^{-7}$. The highest standard deviation is then $\sigma_{\max }=2.0 \times 10^{-6}$ which is a typical value for our dataset (see Fig. 3).

We also test the sensitivity of our method to the wavelet transform entering in the minimisation criterion by comparing the results obtained using two different bi-orthogonal wavelet
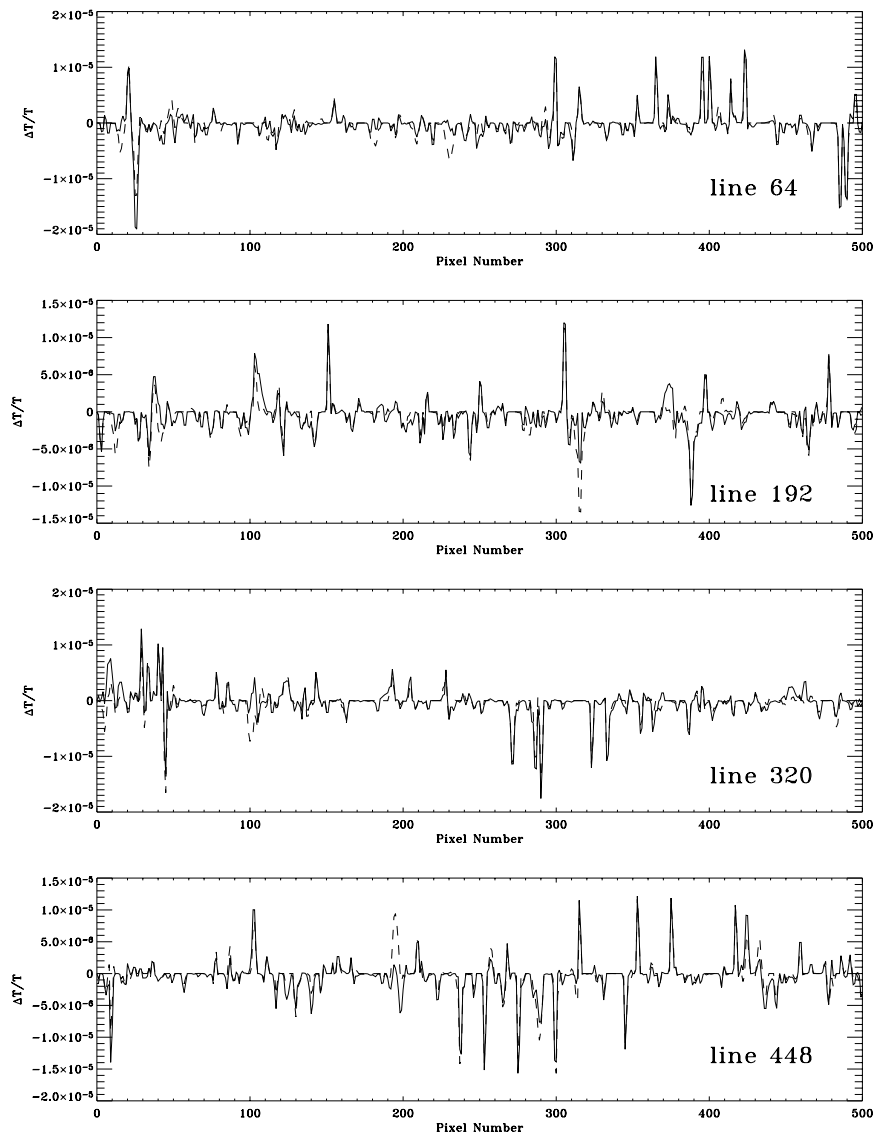

Fig. 6. Cuts across the best reconstructed KSZ map (dashed line) and its original counterpart (solid line). The cuts have the same position in both maps.

bases, the commonly used 9/7 tap filter (Cohen et al. 1990) and the 6/10 tap filter given by Villasenor et al. (1995).

The results for this new set of maps are displayed in Table 2 in terms of the standard deviations of the original and reconstructed KSZ maps, and of the correlation coefficient between the original and reconstructed KSZ maps. We first notice that the results do not depend much on the wavelet basis. As expected, the quality of the reconstruction (given in terms of the correlation coefficient) increases with the standard deviation of the original KSZ map from 0.5 to $\sim 0.8$. The smallest coefficients are obtained for very low standard deviations $\left(<10^{-6}\right)$. For the KSZ map with the lowest standard deviation, the histogram of the reconstructed map (Fig. 8, upper panel, dashed line) shows that the smallest temperature fluctuations are not resolved, which produces an excess of zero values. More generally, the histogram of the reconstructed map behaves like a global envelope to the original histogram (Fig. 8, upper panel, solid line) which does not resolve the details, e.g. the excess of points around $\Delta T / T=1.3 \times 10^{-6}$. In addition, we note an overall rise of the wings of the distribution. Also the power spectrum computed from the reconstructed KSZ map (Fig. 8, middle panel, dashed line) presents an excess of power as compared to the original power spectrum around $\ell=2000$ due to the spatial distribution of the unresolved fluctuations. The latter also causes the lack of power at higher multipoles. This behaviour can also be observed in Fig. 9, which shows a cut 
Table 2. Standard deviations of the KSZ maps and correlation coefficients between original and reconstructed KSZ maps for the same KSZ map with standard deviations ranging from $2.5 \times 10^{-7}$ to $2.0 \times 10^{-6}$. Two wavelet bases are tested.

\begin{tabular}{c|cc|cc}
\hline \hline & \multicolumn{2}{|c|}{$9 / 7$ filter } & \multicolumn{2}{c}{$6 / 10$ filter } \\
Original $\sigma$ & Estimated $\sigma$ & Correlation coefficient & Estimated $\sigma$ & Correlation coefficient \\
\hline $2.5 \times 10^{-7}$ & $2.21 \times 10^{-7}$ & 0.48 & $2.68 \times 10^{-7}$ & 0.45 \\
$3.53 \times 10^{-7}$ & $3.36 \times 10^{-7}$ & 0.54 & $4.02 \times 10^{-7}$ & 0.52 \\
$5.0 \times 10^{-7}$ & $5.52 \times 10^{-7}$ & 0.56 & $5.46 \times 10^{-7}$ & 0.58 \\
$7.07 \times 10^{-7}$ & $8.02 \times 10^{-7}$ & 0.59 & $6.60 \times 10^{-7}$ & 0.67 \\
$1.0 \times 10^{-6}$ & $9.74 \times 10^{-7}$ & 0.68 & $9.16 \times 10^{-7}$ & 0.71 \\
$1.41 \times 10^{-6}$ & $1.35 \times 10^{-6}$ & 0.74 & $1.20 \times 10^{-6}$ & 0.77 \\
$2.0 \times 10^{-6}$ & $1.94 \times 10^{-6}$ & 0.78 & $1.77 \times 10^{-6}$ & 0.81 \\
\hline
\end{tabular}
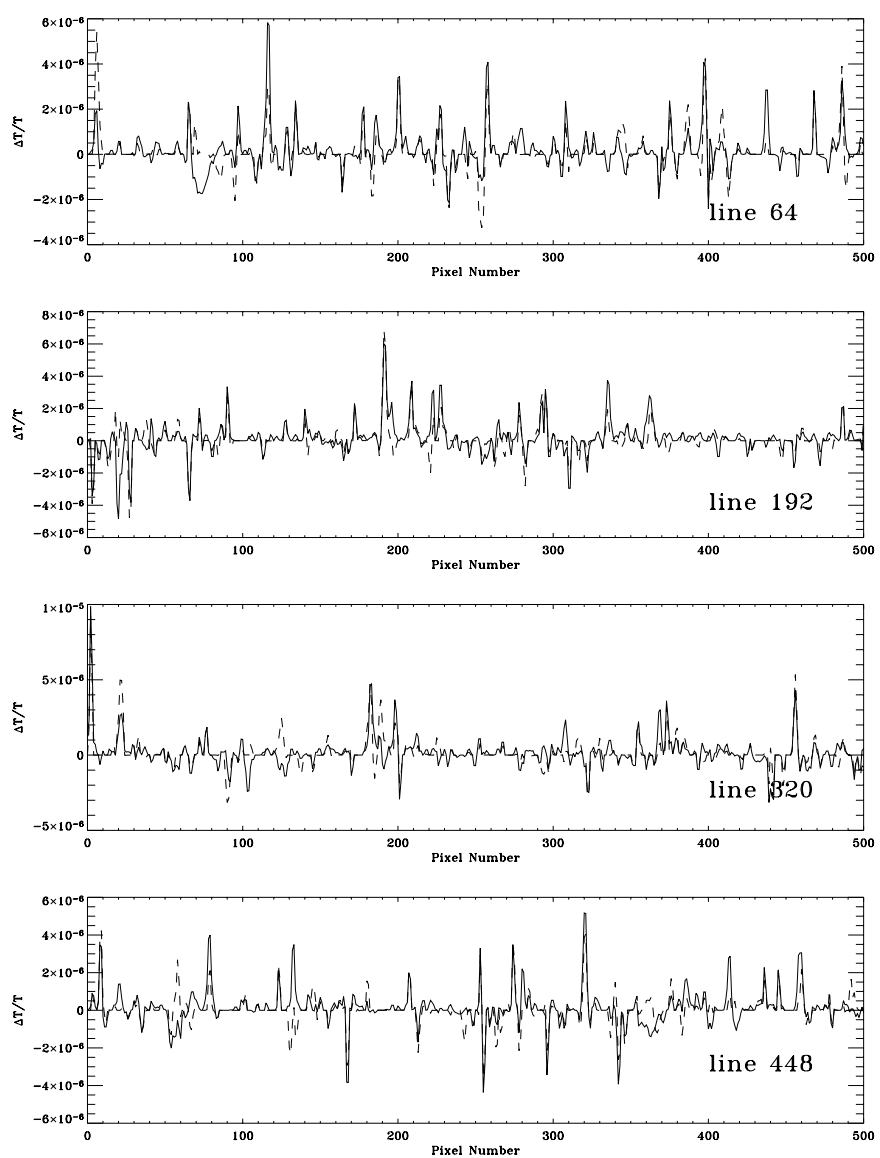

Fig. 7. Same as Fig. 6 for the worst reconstructed KSZ map and its original counterpart.

across the KSZ maps (solid line for the original signal, and dashed line for the reconstructed signal) at the same position but for different standard deviations. It can be noticed that the large scale feature $\sim 50$ arc-min wide at the centre of the cut is poorly resolved due to confusion with the CMB fluctuations. In that worst case, the amplitude of the estimated signal remains, however, proportional to the input signal, but generally the estimation becomes better with increasing standard deviation of the input signal, as it can be seen for the $\sim 10$ arc-min wide fluctuation at the left part of the cut.
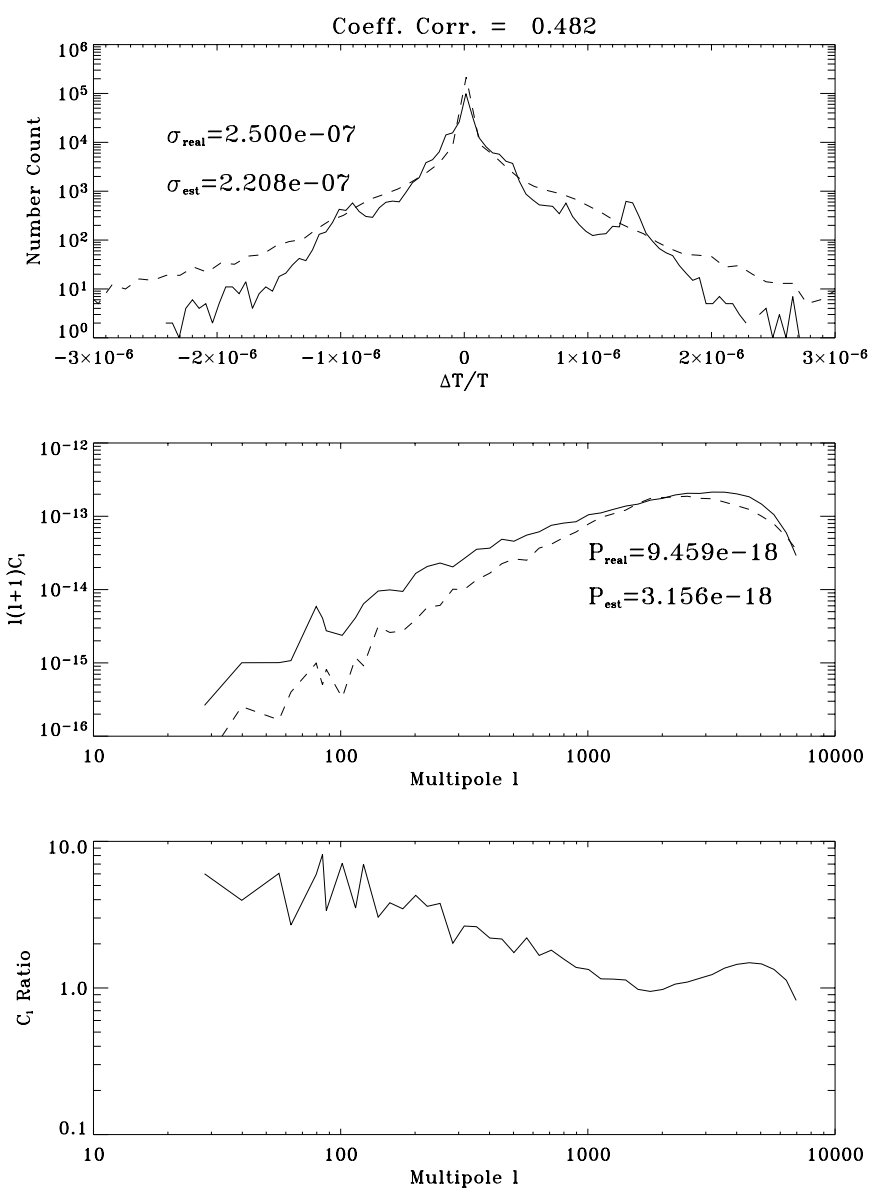

Fig. 8. Top and middle: histogram and power spectrum of the original KSZ map (solid line), and the reconstructed map (dashed line). Bottom panel exhibits the ratio of the two power spectra. The standard deviation of the original map is very low $\left(\sigma_{0}=2.5 \times 10^{-7}\right)$. Note the excess of near zero values in the histogram of the estimated map (logarithmic scale). Note also the very low correlation coefficient 0.48 . This is for the worst case.

\section{Discussion}

We present a method for separating the KSZ signal from primary CMB anisotropies based on two steps: 1) interpolation and 2) reconstruction. Our results clearly depend on the quality of the interpolation used to estimate the primary CMB signal 

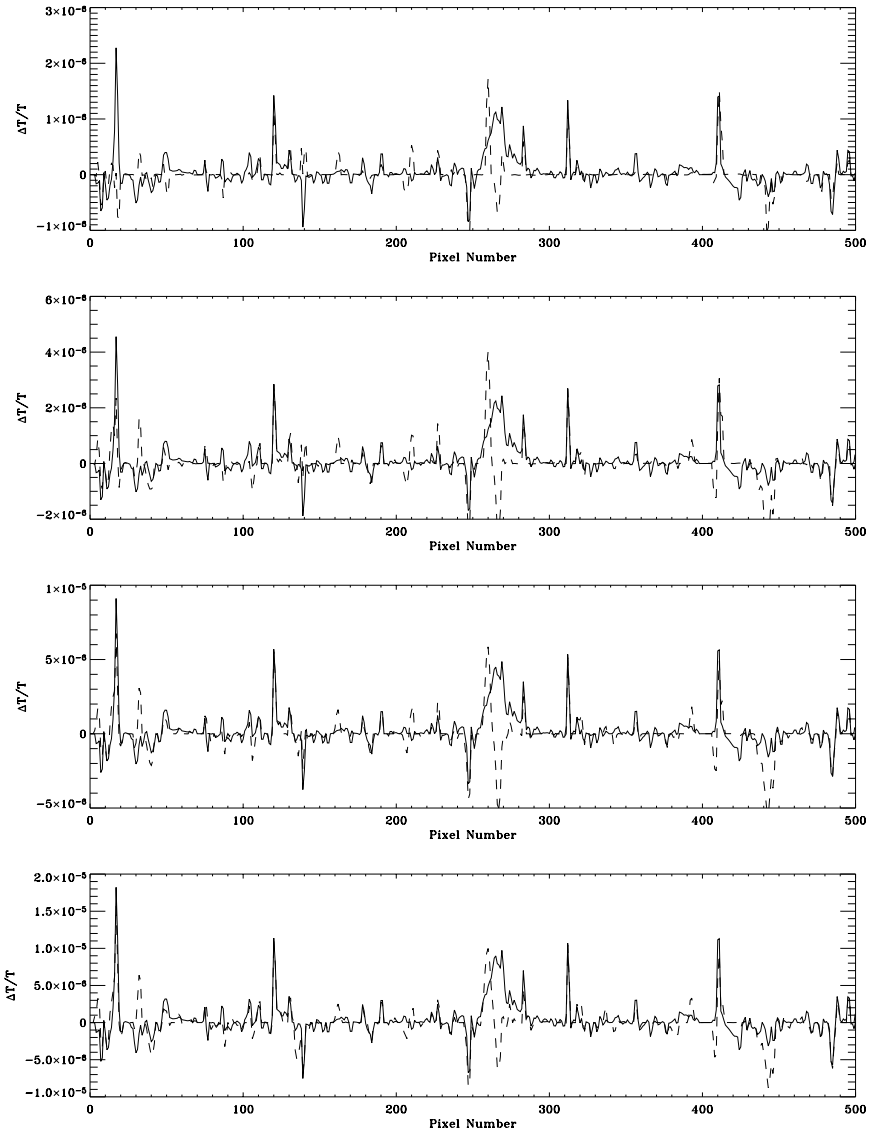

Fig. 9. Cuts across original (solid line) and reconstructed (dashed line) KSZ maps, at the same position, but with increasing (from top to bottom) original standard deviation (for $\sigma: 2.5 \times 10^{-7}, 5.0 \times 10^{-7}$, $1.0 \times 10^{-6}$ and $2.0 \times 10^{-6}$ ). The important difference between original and reconstructed signals in the middle of the cuts illustrates the fact that large scale structures (this one is $\simeq 50$ arc-min) are poorly resolved due to confusion with CMB fluctuations. Note that the largest fluctuation at the left is not reconstructed for the lowest $\sigma$ and that the quality of its reconstruction increases with increasing $\sigma$.

and thus the KSZ maps. In our case this corresponds to the interpolation of a correlated noise, namely the CMB. The results we present in this study seem already very satisfactory but might certainly be improved.

The KSZ reconstruction is based on the set of KSZ estimated maps obtained with a specific choice of TSZ thresholds. We have used here a rather simple but robust method (based on the cumulative distribution function of the pixels in the TSZ map); to determine these thresholds, more sophisticated methods optimising the series of TSZ thresholds need to be investigated.

Using our straightforward choice of thresholds, we have investigated two methods to reconstruct the final KSZ maps: decorrelation and minimisation. The first method is based on the decorrelation approach using the PCA. It significantly underestimates the standard deviations of the reconstructed KSZ maps as compared to the original signal by $50 \%$ on average. More sophisticated decorrelation methods can also be used. Preliminary tests with the Independent Component Analysis (ICA) (Cardoso \& Soulamiac 1993; Hyvärinen 1999) give promising results in terms of the standard deviations. However, the results obtained from the ICA need to be rescaled using external flux constraints which are not always (or easily) available in "real life". For example, in our case we would need to use the fluxes of known clusters to calibrate the reconstructed KSZ maps on the original signal. Despite this limitation, we will continue to investigate this method in the future. The decorrelation method is a blind method whose advantage is that no a priori criteria are needed to obtain the KSZ map. However, the resulting maps are of low quality in terms of standard deviation. The second reconstruction method we use is based on a minimisation technique that takes into account the statistical properties of the KSZ signal, namely: (i) KSZ dominates over the primary anisotropies at small angular scales, and (ii) the KSZ fluctuations follow a non-Gaussian distribution with a non-zero excess kurtosis. In the present study, we use the excess kurtosis of the diagonal wavelet coefficients to characterise the non-Gaussian signatures of the KSZ effect. However, we could generalise the minimisation criterion to include the third moment (skewness) in order to account for, and thus separate, different processes contributing to the signal and having different non-Gaussian characters. The minimisation method we propose gives reconstructed KSZ maps that are in quite good agreement with the original signal with an average correlation coefficient between original and reconstructed KSZ map of 0.78 , and an error of $5 \%$ in the standard deviation of the reconstructed KSZ maps. However, the minimisation method depends greatly on the minimisation criteria and therefore on an a priori knowledge of the reconstructed signal.

The results presented here are based on an ideal case where only the two signals CMB and SZ are taken into account. This simplified test case allows us to investigate the intrinsic limitations of the method. The investigation of noise and additional astrophysical contributions is quite important but it is beyond the scope of our present study. It should a priori be partly treated in the first step component separation (from which we obtain the observables: $y$ and $\delta_{\mathrm{T}}$ maps). However, some contribution from the TSZ signal may remain in the $\delta_{\mathrm{T}}$ map, because of imperfect component separation or when the relativistic corrections to the SZ effect are not corrected for, for example. This will act as an additional and correlated noise. As shown by Diego et al. (2003), this introduces a non-Gaussian signature into the CMB signal and hence errors in the KSZ reconstruction. This non-Gaussian contribution due to the TSZ effect is characterised by a non-zero skewness. We can account for this source of correlated noise and thus correct for it, either at the interpolation stage with the additional constraint that the skewness should be zero (which is the case for the primary CMB anisotropies), or in the minimisation procedure using a generalised criterion including the skewness as well as the excess kurtosis. Another way to overcome this difficulty, is to apply our method to the individual frequency maps and correct for any TSZ spurious contribution. As a matter of fact, the technique we present can be applied to separate TSZ fluctuations from the primary fluctuations. The correlation between two frequency channels, where TSZ dominates, gives indeed a first order spatial template which can be used to obtain the TSZ signal and thus to predict the primary CMB. At this point, 
the first order $y$ map can be used in the next step to better estimate, in an iterative way, the TSZ signal itself. We plan to investigate this method in the future. As for the instrumental noise, it can be taken into account in the interpolation step by relaxing the parameter $\lambda$ (Eq. (2)). However, if the noise is not white then other interpolation methods might have to be used (Sect. 2.1.1 for discussion). Another way to deal with the noise is to minimise not on the non-Gaussian character of the KSZ, but rather on the statistical properties of the remainder (i.e. $\mathrm{CMB}+$ noise + other components) at scales where CMB dominates. We will then obtain an estimate of all the components except KSZ that can then be subtracted from the total signal.

The present work is based on the use of a spatial template to separate KSZ temperature fluctuations from the primary fluctuations. This point has been already noted by Haehnelt \& Tegmark (1996) who used an X-ray emission template to measure the peculiar velocity of clusters. The choice of the spatial template is an important issue for our method since it is used to define the mask and hence the interpolated regions. The spatial template should then be the closest possible to the signal (SZ effect in our case). The optimal choice is really to use the TSZ template itself (similarly to the commonly used matched filter approach). Since SZ traces the intra-cluster gas, we could also use the X-ray emission of clusters as a template. The problem in this case is that the X-ray emission scales with the product $n_{\mathrm{e}}^{2} T_{\mathrm{e}}^{1 / 2}$, whereas the TSZ scales with $n_{\mathrm{e}} T_{\mathrm{e}}$, and consequently the spatial extension of clusters is underestimated if one takes X-ray templates. Additionally, the X-ray observations of galaxy clusters are restricted to a rather small fraction of objects not too distant to suffer from the dimming effect and with high enough intra-cluster temperatures to be detected. The TSZ effect on the contrary is redshift independent and less sensitive to the gas parameters. Moreover, using the TSZ map as a template in our method has the advantage of evaluating the temperature fluctuations (even very low amplitude ones) associated with KSZ in the map without resorting to the knowledge or the measurement of the cluster parameters $\left(n_{\mathrm{e}}, T_{\mathrm{e}}\right)$. The method presented here has proven its success in achieving this goal. In particular, Sect. 3 illustrates how well the KSZ map is reconstructed when the input KSZ signal is decreased by one order of magnitude in terms of standard deviation.

\section{Conclusion}

In this first attempt to extract a map of the KSZ temperature fluctuations from the CMB anisotropies we use a method which is based on very simple and minimal assumptions. We discuss the issue of noise and astrophysical contributions but we do not take them explicitly into account. Therefore, our results show the intrinsic limitations of the method in terms of reconstructing a KSZ map from a mixture of CMB and KSZ anisotropies. We demonstrate that the $15 \mathrm{KSZ}$ reconstructed maps are in quite good agreement with the original input signal with a correlation coefficient between original and reconstructed maps of 0.78 on average, and an error on the standard deviation of the reconstructed KSZ map of only $5 \%$ on average.

To achieve these results, we use the hypothesis that a first step component separation provides us with: (i) a map of Compton parameters for the TSZ effect of galaxy clusters, and (ii) a map of temperature fluctuations for the primary $\mathrm{CMB}+\mathrm{KSZ}$ cluster signal. Our method essentially takes benefit from the spatial correlation between the KSZ and TSZ effects towards the same galaxy clusters. This correlation allows us to use the TSZ map as a spatial template in order to mask, in the CMB + KSZ map, the pixels where the clusters must have imprinted an SZ fluctuation. In practice a series of TSZ threshold is defined, and for each threshold, we estimate the corresponding KSZ signal by interpolating the CMB fluctuations on the masked pixels. The series of estimated KSZ maps finally is used to reconstruct the KSZ map through the minimisation of a criterion taking into account two statistical properties of the KSZ signal (KSZ dominates over the primary anisotropies at small scales, KSZ fluctuations are non-Gaussian distributed).

Acknowledgements. The authors would like to thank Simon Masnou, Jose-Luis Sanz and Philippe Thévenaz for fruitful discussions. We also wish to warmly thank Steen Hansen and Jean-Loup Puget for their helpful comments and an anonymous referee for his/her remarks on an earlier version.

\section{References}

Aghanim, N., De Luca, A., Bouchet, F. R., Gispert, R., \& Puget, J. L. 1997, A\&A, 325, 9

Aghanim, N., \& Forni, O. 1999, A\&A, 347, 409

Aghanim, N., Gorski, K. M., \& Pujet, J.-L. 2001, A\&A, 374, 1

Baccigalupi, C., Bedini, L., Burigana, C., et al. 2000, MNRAS, 318, 769

Benson, B. A., Church, S. E., Ade, P. A., et al. 2003, ApJ, 592, 674

Birkinshaw, M. 1999, Phys. Rep., 310, 97

Bouchet, F. R., \& Gispert, R. 1999, New Astron., 4, 443

Cardoso, J.-F., \& Soulamiac, A. 1993, IEE Proc.-F, 140, 362

Carlstrom, J. E., Holder, G. P., \& Reese, E. D. 2002, ARA\&A, 40, 643

Cayon, L., Martinez-Gonzalez, E., Argueso, F., Banday, A. J., \& Górski, K. M. 2003, MNRAS, 339, 1189

Cohen, A., Daubechies, I., \& Feauveau, J. C. 1990, Technical report, AT\&T Bell Lab., Page:TM 11217-900529-07

Delabrouille, J., Cardoso, J.-F., \& Patanchon, G. 2003, MNRAS, 346, 1089

Diaferio, A., Sunyaev, R. A., \& Nusser, A. 2000, ApJ, 533, 7

Diego, J. M., Hansen, S. H., \& Silk, J. 2003, MNRAS, 338, 796

Faber, S. M., \& Jackson, R. E. 1976, ApJ, 204, 668

Forni, O., \& Aghanim, N. 1999, A\&AS, 137, 553

Haehnelt, M. G., \& Tegmark, M. 1996, MNRAS, 279, 545

Hobson, M. P., Jones, A. W., Lasenby, A. N., \& Bouchet, F. R. 1998, MNRAS, 300, 1

Hobson, M. P., \& McLachlan, C. 2003, MNRAS, 338, 765

Holzapfel, W. L., Ade, P. A. R., Church, S. E., et al. 1997, ApJ, 481, 35

Hyvärinen, A. 1999, IEEE Trans. on Neural Networks, 10, 626

Komatsu, E., Kogut, A., Nolta, M. R., et al. 2003, ApJ, 148, 119

Kuo, C. L., Ade, P. A. R., Bock, J. J., et al. 2004, ApJ, 600, 32

Lamarre, J.-M., et al. 1998, ApJ, 566, 19

Maisinger, K., Hobson, M. P., \& Lasenby, A. N. 2004, MNRAS, 347, 339 
Ostriker, J. P., \& Vishniac, E. T. 1986, ApJ, 306, L51

Press, W. H., Teukolsky, S. A., Vetterling, W. T., \& Flannery, B. P. 1992, Numerical Recipes in FORTRAN: The Art of Scientific Computing (Cambridge University Press)

Rees, M. J., \& Sciama, D. W. 1968, Nature, 511, 611

Reeves, S. J. 1994, IEEE Trans. Image Processing, 3, 319

Rephaeli, Y. 1995, ARA\&A, 445, 33

Santos, M. G., Heavens, A., Balbi, A., et al. 2003, MNRAS, 341, 623

Sorel, M., Aghanim, N., \& Forni, O. 2002, A\&A, 395, 747

da Silva, A. C., Barbosa, D., Liddle, A. R., \& Thomas, P. A. 2001, MNRAS, 326, 155

Sunyaev, R. A., \& Zel'dovich, I. B. 1972, Comments Astrophys. Space Phys., 4, 173

Sunyaev, R. A., \& Zel'dovich, I. B. 1980, ARA\&A, 18, 537

Starck, J. L., Aghanim, N., \& Forni, O. 2004, A\&A, 416, 9
Thévenaz, P., Blu, T., \& Unser, M. 2001, IEEE Trans. on Medical Imaging, 19, 739

Tully, R. B., \& Fisher, J. R. 1977, A\&A, 54, 661

Unser, M., Aldroubi, A., \& Eden M. 1993, IEEE Trans. on Signal Processing, 41, 834

Unser, M. 1995, Proc. of the 1995 IEEE Int. Conf. on Image Processing, 1, 49

Vielva, P., Martinez-Gonzalez, E., Barreiro, R. B., et al. 2003 [arXiv:astro-ph/0310273]

Villasenor, J. D., Belzer, B., \& Liao, J. 1995, IEEE Trans. on Image Processing, 4, 1053

Vishniac, E. T. 1987, ApJ, 322, 597

Wahba, G. 1977, SIAM J. Math. Anal., 14, 651

Wesseling, P. 1992, An Introduction to Multigrid Methods (J. Wiley \& Sons) 